\title{
Machine perfusion of donor heart with normothermic blood versus hypothermic HTK in preserving coronary endothelium in a porcine model of DCD
}

\author{
Pengyu Zhou", Ximao Liu", Zezhou Xiao, Rongning Xu, Xuefeng Lin, Peng Zhu, Jun Lu, Shaoyi Zheng \\ Department of Cardiovascular Surgery, Nanfang Hospital, Southern Medical University, Guangzhou, China \\ Contributions: (I) Conception and design: P Zhou, X Liu S Zheng; (II) Administrative support: P Zhu, J Lu, S Zheng; (III) Provision of study \\ materials or patients: J Lu, S Zheng; (IV) Collection and assembly of data: Z Xiao, R Xu, X Lin; (V) Data analysis and interpretation: P Zhou, X Liu; \\ (VI) Manuscript writing: All authors; (VII) Final approval of manuscript: All authors. \\ \#These authors contributed equally to this work. \\ Correspondence to: Shaoyi Zheng, MD, PhD. Department of Cardiovascular Surgery, Nanfang Hospital, No. 1838 North Guangzhou Avenue, Baiyun \\ District, Guangzhou, China. Email: shaoyizheng@yahoo.com.
}

Background: Both machine perfusion (MP) of donor hearts with autologous blood and crystalloid
perfusates have advantages and disadvantages. Currently, which of the aforementioned preservation
strategies can better preserve the coronary endothelium has not yet been determined. We aim to compare
the impact of hypothermic continuous MP with histidine-tryptophan-ketoglutarate (HTK) solution versus
normothermic continuous MP with autologous blood on coronary endothelium in a porcine ex vivo model
of donation following circulatory death (DCD).
Methods: DCD pigs underwent circulatory arrest via asphyxiation followed by 30 -minute warm ischemia
time. Donor hearts were preserved with either hypothermic MP with HTK solution (MP + HTK group; $4{ }^{\circ} \mathrm{C}$;
$\mathrm{n}=6$ ), or normothermic MP with blood (MP + blood group; $37^{\circ} \mathrm{C}$; $\mathrm{n}=6$ ) for 4 hours. After 2 -hour ex vivo
reperfusion, the assessment of endothelial-dependent (Edep) and -independent (Eind) relaxation of coronary
artery, histopathological analysis, and terminal deoxynucleotidyl transferase-mediated dUTP nick-end
labeling assay were performed. Results: Preservation of DCD hearts with MP + Blood strategy significantly improved both Edep and Eind vasorelaxation of coronary artery compared with MP + HTK strategy (maximum relaxation to bradykinin: MP + HTK $80.9 \% \pm 2.6 \%$ vs. MP + Blood $91.9 \% \pm 1.9 \%, \mathrm{P}<0.001$; maximum relaxation to sodium nitroprusside: MP + HTK $97.1 \% \pm 1.0 \%$ vs. MP + Blood $99.8 \% \pm 0.2 \%, \mathrm{P}<0.05) . \mathrm{MP}+$ Blood strategy significantly decreased nitrotyrosine but increased intercellular adhesion molecule-1 immunoreactivity in the coronary artery. The number of TUNEL-positive cells in MP + Blood group were significantly fewer compared with MP + HTK group.

Conclusions: Compared with MP + HTK strategy, MP + Blood strategy significantly alleviates coronary endothelial dysfunction during donor heart preservation. This protective effect is associated with the inhibition of apoptosis and nitro-oxidative stress in coronary artery.

Keywords: Machine perfusion; heart preservation; donation following circulatory death; coronary artery; endothelium; porcine

Submitted Jan 08, 2020. Accepted for publication Jun 03, 2020.

doi: $10.21037 / \mathrm{apm}-20-131$

View this article at: http://dx.doi.org/10.21037/apm-20-131 


\section{Introduction}

The discrepancy between the demand and supply of donor hearts continues to limit the number of heart transplantation (HTX) performed worldwide (1). The use of hearts obtained with donation after circulatory death (DCD) is one of the most promising approaches to increase the availability of donor hearts (2). Although irreversible global warm ischemia/reperfusion (I/R) injury suffered by DCD hearts remains a legitimate concern (3), more than 70 DCD heart transplantations have been performed since 2014 (4). In the meantime, the mortality rate of those patients on the waiting list for HTX can be reduced by $40 \%$ (5). Furthermore, HTX from DCD hearts provides comparable 1 -year survival rates and short-term outcomes compared to traditional HTX from donor hearts of donation after brain death (6).

On the other hand, coronary endothelium holds a critical position in the regulation of coronary blood flow, the maintenance of post-transplant cardiac function, complement activation, platelet aggregation, modulation of the inflammatory response associated with I/R injury (7) and the development of late events (e.g., graft rejection and postoperative cardiac allograft vasculopathy) (8). However, during the perioperative management of HTX in DCD hearts, I/R injury is an inevitable pathological process which causes endothelial dysfunction of the coronary artery. Prolonged warm ischemia time can lead to both impaired endothelial-independent and endothelial-dependent vasodilation of the coronary artery by increasing endothelial nitric oxide synthase (eNOS) uncoupling in an isolated working rat heart model of DCD (9). In addition, the following reperfusion can result in high production of reactive oxygen species (ROS), the generation of inflammatory cytokines and infiltrating neutrophil (10), and the development of acidosis due to anaerobic metabolism (11). Therefore, the preservation of coronary endothelium should be as significant as myocardium in the preservation of DCD hearts (12).

Currently, DCD hearts were utilized successfully by warm blood perfusion preservation using the Transmedics Organ Care System in the clinical setting (13). In addition, a series of previous preclinical studies demonstrated that compared with static cold storage, hypothermic continuous machine perfusion (MP) resulted in improved preservation of energy charge and functional recovery of DCD hearts grafts (14-17). However, no experimental studies compared the effect of hypothermic continuous MP with oxygenated crystalloid perfusate and normothermic continuous MP with oxygenated warm blood on the endothelial function of the coronary artery of donor hearts.

Therefore, the present study aims to investigate the impact of hypothermic continuous MP with histidinetryptophan-ketoglutarate (HTK) solution versus normothermic continuous MP with warm blood on the coronary endothelium of donor hearts in a porcine ex vivo model of donation following circulatory death. We present the following article in accordance with the ARRIVE reporting checklist (available at http://dx.doi.org/10.21037/ apm-20-131).

\section{Methods}

\section{Animals}

All the healthy male pigs weighing 30-35 kg were used in this experiment and received care in compliance with the Guide for the Care and Use of Laboratory Animals (National Institutes of Health Publication No. 85-23, revised 1996). The study was approved by institutional ethics committee of Nanfang Hospital of Southern Medical University (No. NFEC-2018-056).

The pigs were housed in large hog pens $(n=3$ per hog pen) at constant ambient temperature $\left(22 \pm 2{ }^{\circ} \mathrm{C}\right)$ in lightcontrolled rooms (12-12 h light-dark cycles) and were given food and water access ad libitum.

\section{DCD beart procurement}

An intramuscular injection of tiletamine $(2.4 \mathrm{mg} / \mathrm{kg})$, zolazepam $(2.4 \mathrm{mg} / \mathrm{kg})$, and xylazine $(0.9 \mathrm{mg} / \mathrm{kg})$ was used to sedate all the pigs weighing between 30 and $35 \mathrm{~kg}$. Orotracheal intubation was performed and general anesthesia was maintained with $2-3 \%$ isoflurane. We performed a median sternotomy and delivered 1,000 units (U)/kg of heparin intravenously. To monitor the central venous and aortic pressures, we placed $7 \mathrm{~F}$ and $6 \mathrm{~F}$ catheters into the internal jugular vein and common carotid artery, respectively. Mechanical ventilation was ceased and the endotracheal tube was disconnected. Circulatory arrest time was defined as the time when mean arterial pressure was equal to central venous pressure. Warm ischemia time was defined as the duration from the withdrawal of ventilator support to the administration of preservation solution. All the pigs underwent 30-minute warm ischemia time. 
Immediately before the end of 30-minute warm ischemia time, cannulas were inserted into the right atrium (for blood collection) and aortic root (for HTK solution flush). Blood (1 L) was drained from the animal just before aortic cross-clamp application, and HTK solution was administered combined with topical cooling at the end of 30-minute warm ischemia time. Then rapid cardiectomy was performed.

\section{The preservation of heart grafts}

The heart grafts were preserved for 4 hours, each randomly assigned to either the MP with HTK solution (Custodiol ${ }^{\circ}$, Dr. F. Köhler Chemie GmbH, Bensheim, Germany) (MP + HTK group, $n=6)$ or blood (MP + Blood group, $n=6)$ preservation strategies.

A roller pump (Stockert Instrumente GmbH, Munich, Germany), a Medtronic Affinity NT oxygenator (Medtronic, Minneapolis, USA), Lilliput I hard-shell venous reservoir (Sorin Group, Mirandola, Italy), leukocyte filter (Pall Corporation, New York, USA), and a heater-cooler unit were used to construct the home-made ex vivo perfusion circuit.

In the MP + HTK group, the heart grafts were perfused on the ex vivo perfusion circuit with 1 L HTK solution for 4 hours. The heart grafts were suspended by the brachiocephalic trunk and retrograde aortic perfusion was performed. The temperature was maintained constantly at $4{ }^{\circ} \mathrm{C}$ with an external heater-cooler unit. The perfusion pressure was $15 \mathrm{mmHg}$. A partial oxygen pressure of 150 $200 \mathrm{mmHg}$ was maintained in the HTK solution under a gas phase of $95 \%$ oxygen and $5 \%$ carbon dioxide.

In the MP + Blood group, the heart grafts were perfused on the ex vivo perfusion circuit with $1 \mathrm{~L}$ autologous blood with $500 \mathrm{~mL}$ of Gelofusine, heparin (1,000 IU/L), glucose (2 $\mathrm{g} / \mathrm{L})$, insulin $(50 \mathrm{IU} / \mathrm{L})$, aspartate $(14 \mathrm{mmol} / \mathrm{L})$, bicarbonate $(20 \mathrm{mmol} / \mathrm{L})$, and adenosine $(1 \mathrm{mg} / \mathrm{L})$. Temperature was maintained constantly at $37^{\circ} \mathrm{C}$ with an external heater-cooler unit. The perfusion pressure was $60 \mathrm{mmHg}$. A partial oxygen pressure of $150-200 \mathrm{mmHg}$ was maintained in the autologous blood under a gas phase of $95 \%$ oxygen and $5 \%$ carbon dioxide.

\section{Reperfusion protocol}

After 4-hour machine preservation with HTK solution or blood, the grafts were reperfused for 2 hours on the ex vivo perfusion circuit with the reperfusion protocol of MP +
Blood strategy.

\section{The harvest and preparation of coronary artery of heart graft}

After 2-hour reperfusion, the proximal part of left anterior descending coronary artery (LAD) was explanted from the heart graft and immediately placed in cold $\left(4^{\circ} \mathrm{C}\right)$ KrebsHenseleit solution (KHL) treated with 95\% oxygen-5\% carbon dioxide. Using an operation microscope, the LAD was dissected free from the adhering fat and connective tissue without damaging the endothelium and cut into several segments (each 4-mm in length).

\section{In vitro organ bath experiment}

The coronary artery rings were stretched to $3.0 \mathrm{~g}$ and allowed to stabilize for 90 minutes. After the equilibration period, they were then precontracted with a thromboxane A2 analog, U46619 compound $\left(5 \times 10^{-7} \mathrm{M}\right)$ until a stable plateau was reached. The Edep vasorelaxation of the coronary artery was assessed by the addition of cumulative concentrations of bradykinin (BK) $\left(10^{-9}-5 \times 10^{-6} \mathrm{M}\right)$. Similarly, sodium nitroprusside (SNP) $\left(10^{-9}-10^{-4} \mathrm{M}\right)$ was applied to assess the Eind vasorelaxation of the coronary artery. Contractile responses to U46619 were described in grams of tension. Edep and Eind vasorelaxation [and its maximum $\left(\mathrm{R}_{\max }\right)$ ] were expressed as the percentage of contraction induced by $\mathrm{U} 46619$. Half-maximum response $\left(\mathrm{EC}_{50}\right)$ values were generated from individual concentration-responses by fitting experimental data to a sigmoidal equation using Origin 7.0 (Microcal Software Northampton, MA, USA). The sensitivity to BK and SNP was assessed by negative logarithm of the corresponding $\mathrm{EC}_{50}\left[\mathrm{pD}_{2}=-\log \mathrm{EC}_{50}(\mathrm{M})\right]$.

\section{Immunobistochemical stainings}

Distal regions of the LAD were fixed in buffered paraformaldehyde solution (4\%) immediately after harvesting and embedded in paraffin. Then, 4- $\mu \mathrm{m}$ thick sections were placed on adhesive slides. The immunoreactivity to nitrotyrosine (1:100; MilliporeSigma, Burlington, USA), ICAM-1 (intercellular adhesion molecule-1; 1:100; Novus Biologicals, Colorado, USA), caspase-3 (1:1,000; Active and Cleaved, Novus Biologicals, Colorado, USA), eNOS (endothelial nitric oxide synthase 1:100; Novus Biologicals, Littleton, CO, USA) was tested. Semi-quantitative analysis for the expression of 
Table 1 Quantitative analysis of the vascular function of the coronary artery

\begin{tabular}{|c|c|c|c|}
\hline Value & \multicolumn{3}{|c|}{ Group } \\
\hline Maximal contraction to $U 46619, \mathrm{~g}$ & $9.2 \pm 1.0$ & $15.3 \pm 1.4^{*}$ & 0.0012 \\
\hline Rmax to $\mathrm{BK}, \%$ & $80.9 \pm 2.6$ & $91.9 \pm 1.9^{\#}$ & 0.0006 \\
\hline $\mathrm{pD} 2$ to $\mathrm{BK}$ & $7.4 \pm 0.3$ & $8.0 \pm 0.1$ & 0.4774 \\
\hline pD2 to SNP & $6.3 \pm 0.1$ & $6.5 \pm 0.1$ & 0.1694 \\
\hline
\end{tabular}

${ }^{*} \mathrm{P}<0.05$ versus MP + HTK. ${ }^{\#} \mathrm{P}<0.001$ versus MP + HTK. N, number of animals; $n$, number of coronary aortic rings; MP, machine perfusion;

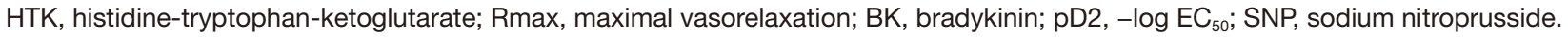

protein was performed by 2 blinded observers based on staining intensity and the distribution of the labeled target protein. The evaluation was done in four randomized nonoverlapping fields of the $\mathrm{LAD}$, and the average value was calculated for the statistical analysis.

\section{Terminal deoxynucleotidyl transferase-mediated dUTP nick-end labeling reaction}

The terminal deoxynucleotidyl transferase-mediated dUTP nick-end labeling (TUNEL) assay was performed to detect DNA strand breaks in LAD. Rehydrated tissue sections were treated with $20 \mu \mathrm{g} / \mathrm{mL}$ of Proteinase K (SigmaeAldrich, Seelze, Germany, Sigma-Aldrich, St. Louis, MO, USA) to retrieve antigenic epitopes following the protocol of the commercial kit (Roche, Mannheim, Germany, Roche, Basel, Switzerland). Tissue sections were incubated with TUNEL reaction mixture in a humidified atmosphere for one hour in the dark at $37^{\circ} \mathrm{C}$. At last, cell nucleis of the tissue sections were counterstained with 4', 6-diamidino-2-phenylindole (DAPI). Images were taken using a fluorescent microscope (Olympus IX81; Olympus).

\section{Statistical analysis}

The results were expressed as mean \pm standard error of the mean (SEM). Statistical analysis was performed with GraphPad Prism 7.02 software (GraphPad Sofware, Inc., CA, USA). Data was tested for normality using the ShapiroWilk test before statistical tests. The two-sample student t-test was conducted to compare the means of MP + HTK and MP + Blood groups from normally distributed data. If data was not normally distributed, then a nonparametric
Mann-Whitney test was applied. A value of $\mathrm{P}<0.05$ was considered statistically significant.

\section{Results}

\section{Vasomotor function}

After 2-hour reperfusion, the preservation of DCD hearts using the MP + Blood strategy significantly increased maximum contraction to U46619 of coronary artery compared with the MP + HTK strategy $(\mathrm{P}<0.05)$ (Table 1, Figure $1 A$ ). The preservation of DCD hearts with the MP + Blood strategy significantly improved both Rmax (maximal vasorelaxation) for Edep $(\mathrm{P}<0.001)$ and Eind $(\mathrm{P}<0.05)$ vascular relaxation of coronary artery compared with the MP + HTK strategy (Table 1, Figure 1B,C). Although not statistically different ( $\mathrm{pD} 2$ to $\mathrm{BK}=0.4774 ; \mathrm{pD} 2$ to SNP $=0.1694)$, the concentration-response curves to both BK and SNP in the MP + Blood group showed a tendency towards shifting to left compared with the MP + HTK group, indicating the preservation of DCD hearts using $\mathrm{MP}+$ Blood strategy conferred a tendency to improve the sensitivity of coronary artery to both BK and SNP (Table 1, Figure 1D,E).

\section{Nitro-oxidative stress in the coronary artery of the donor bearts}

To evaluate the level of nitro-oxidative stress in LAD in both groups, we assessed the immunoreactivity of nitrotyrosine, a marker of peroxynitrite-mediated damage. Immunostaining for nitrotyrosine showed that the preservation of DCD hearts using the MP + Blood strategy 

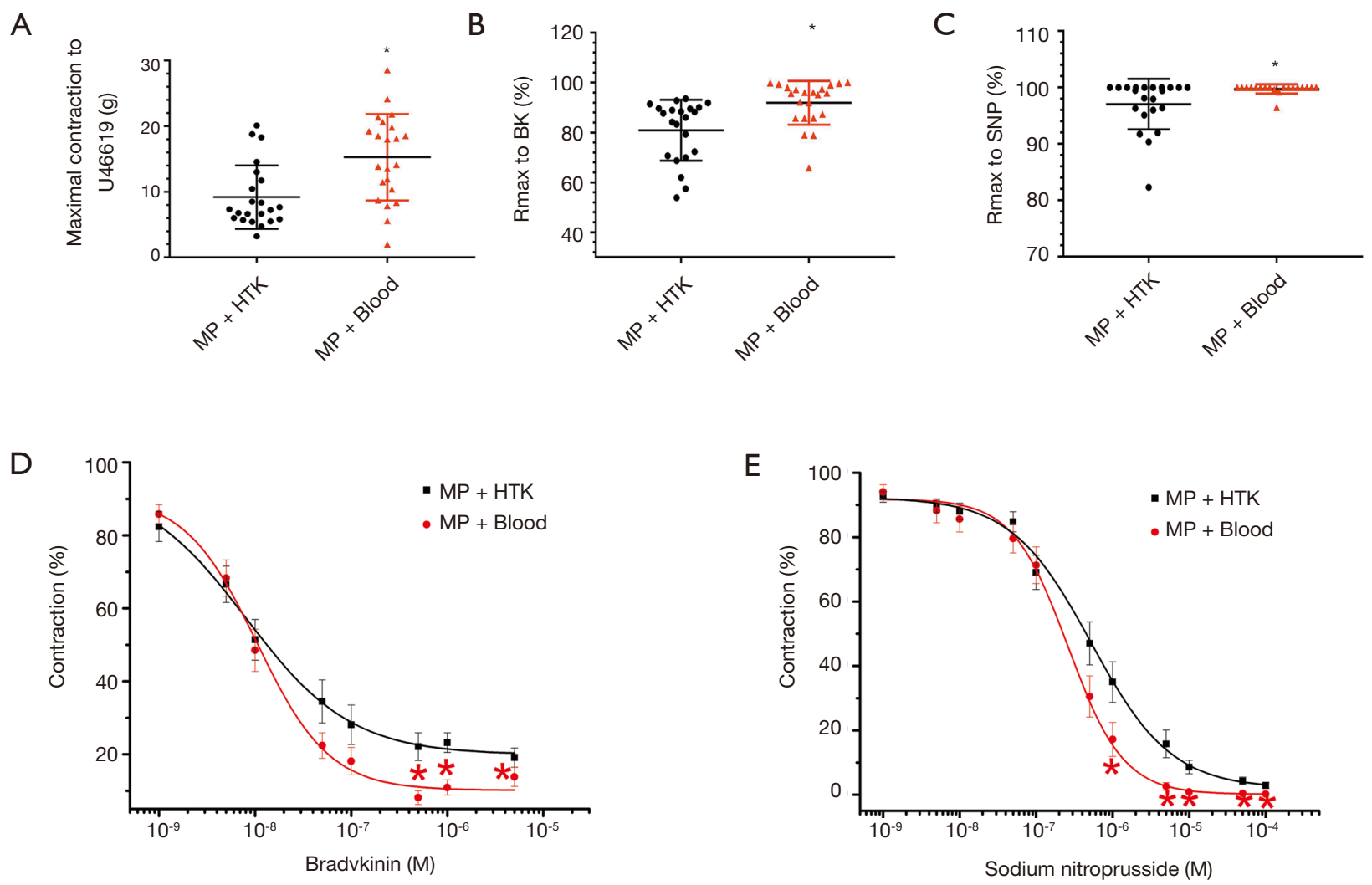

Figure 1 The effect of different machine perfusion strategies on contractile and relaxant responses in the coronary artery. (A) Maximum vasoconstriction in response to $\mathrm{U} 46619$ ( $\mathrm{N}=6$ animals, $\mathrm{n}=22$ coronary aortic rings for each group). (B) Maximum vasorelaxation in response to bradykinin (BK) ( $\mathrm{N}=6$ animals, $\mathrm{n}=22$ coronary aortic rings for each group). (C) Maximum vasorelaxation in response to sodium nitroprusside (SNP) (N=6 animals, $\mathrm{n}=22$ coronary aortic rings for each group). (D) Endothelial-dependent (Edep) vasorelaxation (N=6 animals, $\mathrm{n}=22$ coronary aortic rings for each group). (E) Endothelial-independent (Eind) vasorelaxation ( $\mathrm{N}=6$ animals, $\mathrm{n}=22$ coronary aortic rings for each group). Data is represented as mean \pm SEM. * $\mathrm{P}<0.05$ vs. MP + HTK. MP, machine perfusion; HTK, histidine-tryptophanketoglutarate.

significantly lowered the immunoreactivity of nitrotyrosine in the wall of LAD compared with the MP + HTK strategy (Figure 2A).

\section{The expression of ICAM-1 and eNOS in the coronary artery of the donor hearts}

ICAM-1 is regarded as one of the adhesion molecules which can lead to the recruitment of leukocytes to the endothelium and their migration into the tissue. Immunohistochemical staining revealed significantly higher expression of ICAM-1 in LAD of the MP + Blood group compared with the MP + HTK group (Figure 2B).
eNOS is an important synthase which can convert $\mathrm{L}$-arginine to nitric oxide (NO), thereby regulating the endothelial function. Immunostaining analysis showed that no significant difference in the expression of eNOS in the coronary artery was found between both groups (Figure 2C).

\section{Apoptosis in the coronary artery of the donor hearts}

Immunohistochemical analysis demonstrated that no significant change was found in the vessel wall of LAD for caspase-3 staining between both groups (Figure 2D). An increased density of TUNEL-positive nuclei indicates severe DNA fragmentation in the arterial wall (intima and 

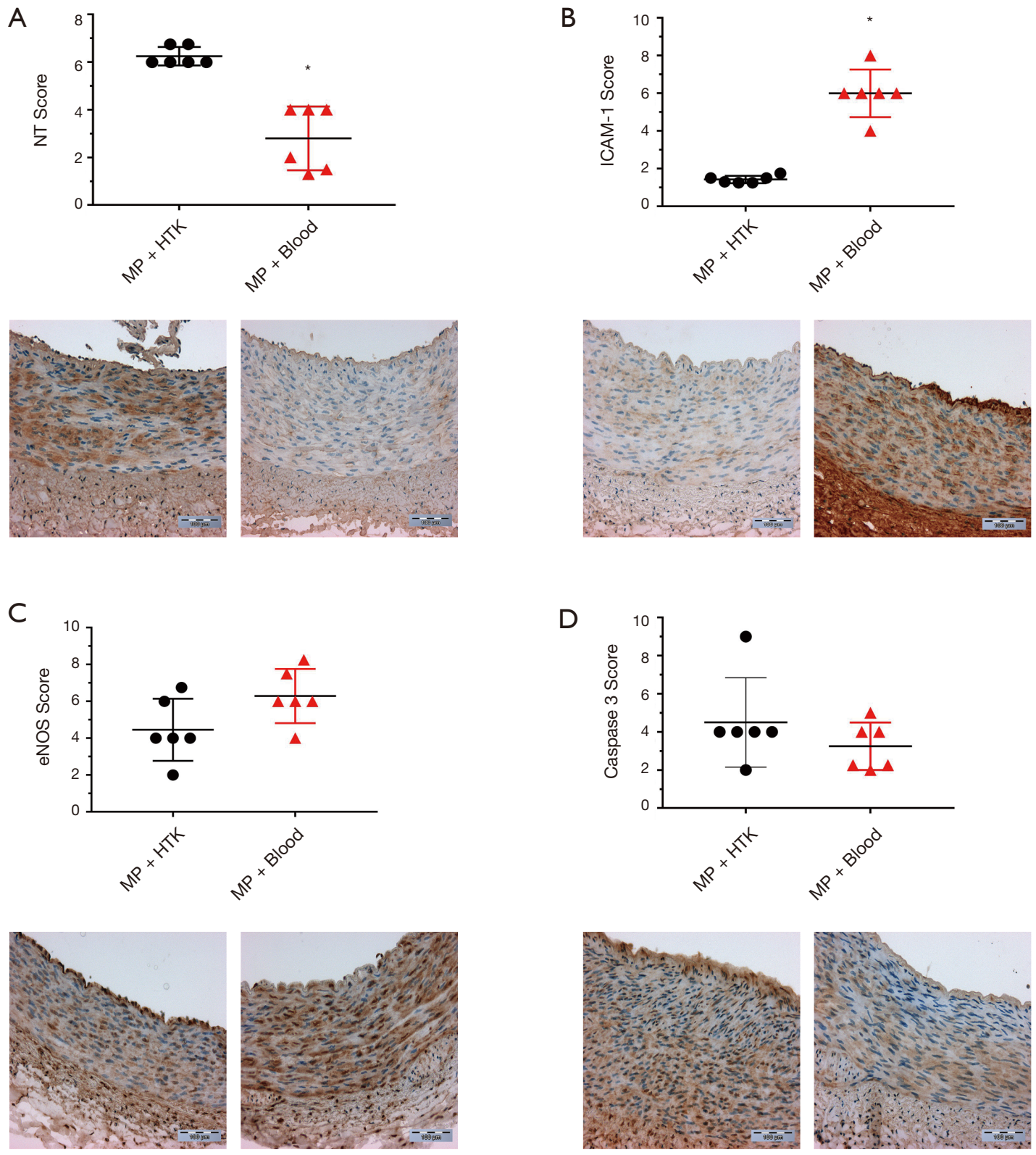

Figure 2 The Effect of different machine perfusion strategies on the expression of nitrotyrosine, ICAM-1, eNOS and caspase-3 in the coronary artery. Representative photomicrographs and immunohistochemical scores of stainings (brown staining) for (A) nitrotyrosine (NT, ×200 magnification, scale bar: $100 \mu \mathrm{m}, \mathrm{N}=6$ animals), (B) ICAM-1 (×200 magnification, scale bar: $100 \mu \mathrm{m},(\mathrm{C})$ eNOS (×200 magnification, scale bar: $100 \mu \mathrm{m}, \mathrm{N}=6$ animals), and (D) caspase 3 ( $\times 200$ magnification, scale bar: $100 \mu \mathrm{m}, \mathrm{N}=6$ animals). Data is represented as mean \pm SEM. *, $\mathrm{P}<0.05$ vs. MP + HTK. NT, nitrotyrosine; MP, machine perfusion; HTK, histidine-tryptophan-ketoglutarate; ICAM-1, intercellular adhesion molecule-1; eNOS, endothelial nitric oxide synthase. 
A
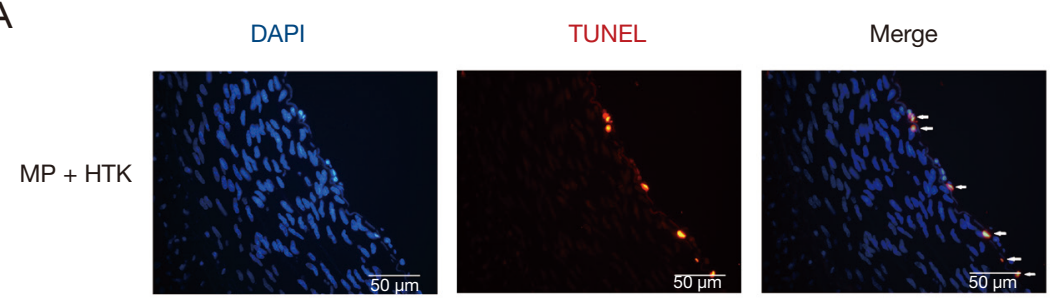

B
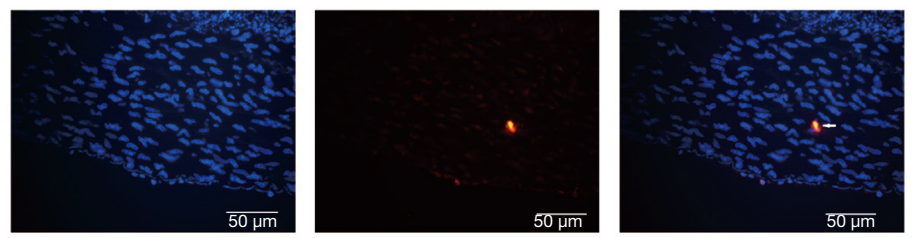

C

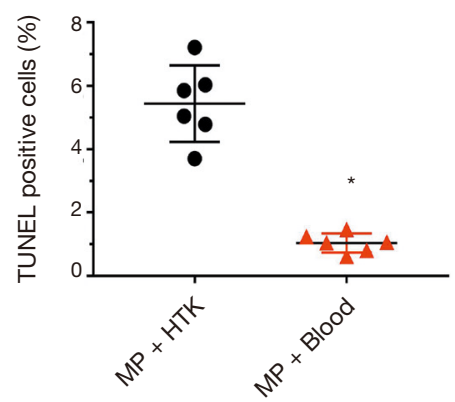

Figure 3 The effect of different machine perfusion strategies on apoptosis in the coronary artery. (A and B) Representative photomicrographs of immunohistochemical stainings for terminal deoxynucleotidyl transferase-mediated dUTP nick end-labeling (TUNEL). (C) Quantitation of apoptotic cells ( $\mathrm{N}=6$ animals). White arrows indicate TUNEL ${ }^{+}$cells. Scale bar: $100 \mu \mathrm{m}$. Data is represented as mean \pm SEM. * $\mathrm{P}<0.05$ vs. MP + HTK. MP, machine perfusion; HTK, histidine-tryptophan-ketoglutarate.

media) in the experimental groups. TUNEL-positive cells in the MP + Blood group were significantly fewer compared with the MP + HTK group (Figure $3 A, B, C$ ).

\section{Discussion}

In the present study, we have demonstrated that although compared with MP + HTK strategy, MP + Blood preservation protocol significantly upregulates the expression of ICAM-1 in the coronary artery, it significantly alleviates endothelial dysfunction of coronary artery of donor hearts in a porcine ex vivo model of DCD. This protective effect is associated with the inhibition of apoptosis and nitro-oxidative stress in the coronary artery.

During the perioperative management of HTX with DCD hearts, I/R injury can lead to inevitable endothelial dysfunction of the coronary artery. Méndez-Carmona et al. demonstrated that endothelial-dependent vasodilation of coronary artery was significantly impaired compared with non-ischemic hearts with $\geq 24$ minutes of warm ischemia, whereas endothelial-independent vasodilation was impaired with $\geq 27$ minutes of warm ischemia in an isolated working rat heart model of DCD (9). In addition, the following reperfusion can lead to a burst of ROS to the luminal surface of the blood vessel, thereby causing deleterious effect on the endothelial function of the coronary artery (18). Tsao and Lefer (19) showed that endothelial dysfunction could be present as early as 2.5 minutes after the onset of reperfusion. Therefore, under the context of donor heart preservation, the effect of different preservation and storage techniques on the coronary endothelium submitted to I/R injury needs to be brought into focus $(8,12)$. 


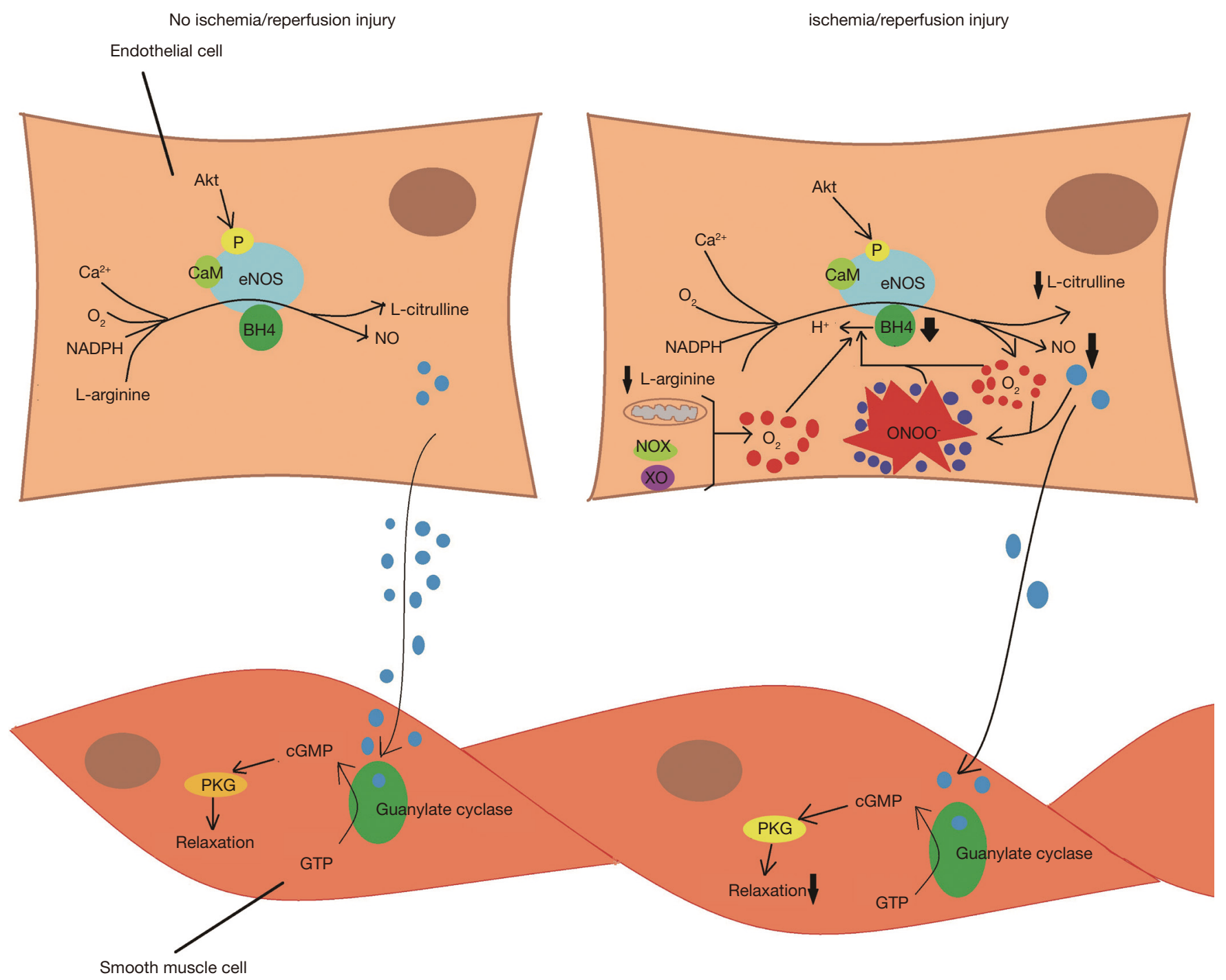

Figure 4 Schematic showing the mechanisms of how ischemia/reperfusion injury induces endothelial dysfunction in endothelial cells. (A) Under physiologic conditions, eNOS-mediated vasodilation can be operated normally. (B) Under global ischemia and reperfusion, eNOS uncoupling and peroxynitrite formation decrease the bioavailability of NO, thereby resulting in endothelial dysfunction in endothelial cells. Akt, protein kinase B; BH4, tetrahydrobiopterin; $\mathrm{Ca}^{+}$, calcium; CaM, calmodulin; cGMP, cyclic guanosine monophosphate; eNOS, endothelial nitric oxide synthase; GTP, guanosine triphosphate; $\mathrm{H}+$, hydrogen; NADPH, nicotinamide adenine dinucleotide phosphate; $\mathrm{NO}$, nitric oxide; $\mathrm{NOX}$, NADPH oxidases; $\mathrm{O}_{2}$, oxygen; $\mathrm{O}_{2}^{-}$, superoxide; $\mathrm{ONOO}^{-}$, peroxynitrite; $\mathrm{P}$, phosphorylation; $\mathrm{PKG}$, protein kinase G; $\mathrm{XO}^{-}$, xanthine oxidase.

In the present study, we found that compared with MP + HTK strategy, the preservation of DCD hearts with the MP + Blood strategy significantly increased maximal vasoconstriction of the coronary artery to U46619, improved endothelial-dependent and endothelialindependent vasorelaxation of the coronary artery following I/R injury. Endothelial function depends on the availability of NO, which is mainly generated by eNOS. Under physiologic conditions, eNOS can convert L-arginine to $\mathrm{NO}$ and L-citrulline in the existence of nicotinamide adenine dinucleotide phosphate (NADPH), flavin mononucleotide, flavin adenine dinucleotide, and $\mathrm{O}_{2}$, and the redox-sensitive cofactor, tetrahydrobiopterin (BH4) (20) (Figure 4). In line with this concept, we revealed that the 
MP + Blood strategy significantly alleviated endothelial dysfunction by improving I/R injury-induced impaired NO production, as determined by BK-induced vasodilatory responses.

In the current study, although significantly improved endothelial-dependent relaxation could be seen in the MP + Blood strategy compared with the MP + HTK strategy, no significant difference was found in the expression of eNOS between both groups. The endothelial-dependent vasodilation of the coronary artery that we observed did not result from alterations in the expression of eNOS. This may not be surprising since $\mathrm{NO}$ production can be regulated by several mechanisms, including changes in the expression and activity of eNOS, the levels of calcium and oxygen, or the availability of substrates and cofactors.

During I/R injury, endothelial dysfunction likely resulted from eNOS uncoupling. The appearance of ROS can uncouple eNOS through the oxidation of $\mathrm{BH} 4$, which reduces the bioavailability of NO. The uncoupled eNOS can result in the production of $\mathrm{O}_{2}^{-}$instead of $\mathrm{NO}$ (20-23). Moreover, $\mathrm{O}_{2}^{-}$can react with $\mathrm{NO}$ to produce $\mathrm{ONOO}^{-}$, which further leads to the oxidation of $\mathrm{BH} 4$, thereby aggravating eNOS uncoupling and becoming a vicious circle (Figure 4). Nitrotyrosine in proteins is the detectable marker for indirectly detecting the formation of $\mathrm{ONOO}^{-}$. In the current study, we found that the MP + Blood strategy significantly reduced the expression of nitrotyrosine, indicating that it reduced the level of $\mathrm{ONOO}^{-}$and nitrooxidative stress, and increased NO availability, which occurred in parallel with the improved endothelial function of the coronary artery. Although the coronary endothelium suffered a burst of ROS by twice reperfusions in the MP + Blood protocol compared with the MP + HTK strategy, blood perfusion with erythrocytes had some advantages, including anti-oxidant properties, the regulation of vascular tone and highly effective oxygen transport in comparison with crystalloid perfusates (24).

In addition, the formation of $\mathrm{ONOO}^{-}$can result in the oxidation of lipids, sulfhydrils, DNA, and redox molecules, as well as the damage of critical proteins for cell survival and the nitration of protein tyrosine residues (20). In line with this perspective, our TUNEL assay demonstrated that more apoptotic cells in the coronary artery were found in the MP + Blood group.

Reperfusion can lead to the upregulation of adhesion molecules, such as vascular cell adhesion protein 1 (VCAM1), ICAM-1, thereby resulting in the recruitment of leukocytes to the endothelium (25). In the current study, we reported a significant overexpression of ICAM-1 in vascular graft in the MP + Blood group. This finding might be due to twice reperfusions, high level of free fatty acids (26), lactates of donor blood and low $\mathrm{pH}$ environment (27) in blood perfusion, which upregulated the expression of ICAM-1 and the release of inflammatory cytokines by leukocytes $(28,29)$.

Notably, although we demonstrated that MP + Blood strategy significantly alleviated endothelial dysfunction of coronary artery of donor hearts in a porcine ex vivo DCD model compared with the MP + HTK strategy, both of blood perfusion and crystalloid perfusates had their own positive and negative effects on the coronary endothelium. With erythrocytes, blood perfusion has some advantages, such as anti-oxidant properties, the regulation of vascular tone and highly effective oxygen transport (24). However, blood reperfusion is associated with some disadvantages, including twice reperfusions, which induce the release of ROS and inflammatory cytokines $(28,29)$. Moreover, donor blood used for perfusion may have a high level of catecholamines, lactate, free fatty acids (26), and low $\mathrm{pH}$ environment (27), which could induce deleterious effects on the endothelial function of the coronary artery. In the MP with crystalloid perfusates strategy, we can precisely control conditions and perfusate composition, while this preservation technique can lead to a higher level of edema of vascular graft than blood perfusate $(30,31)$. From the perspective of translational medicine, the use of machine perfusion of donor heart with normothermic blood can increase the transplant volumes by $15-20 \%$ with successful utilization of donor hearts retrieved from higher risk DBD and donation after circulatory death donors $(6,13,32)$. Furthermore, the machine perfusion of donor hearts not only allows us to ex vivo assess, preserve and repair the grafts, but also provides a unique platform for the administration of the extracellular vesicles and exosome of mesenchymal stem cells to strengthen the cardioprotective effect of donor heart (33). Therefore, machine perfusion with normothermic blood could become a promising and novel alternative for the preservation of donor heart in the future. The present study should be interpreted with caution due to the limitations of several restraints. First, reperfusion of donor hearts was performed in vitro on a Langendorff apparatus instead of in vivo transplantation, although numerous confounding factors in a transplantation model can be avoided, recirculating donor blood from DCD 
hears with inflammatory cytokines can potentially impact the coronary endothelium. Secondly, our model of DCD hearts still differed from clinical reality. In our protocol, hearts were left untouched in the donor for around 25min warm ischemia time regardless of time to circulatory cessation. While in most of the clinical settings, a standoff period is only 5 minutes after death, which is determined by the cessation of circulation. Therefore, DCD hearts in the present study suffered a longer and more severe warm ischemia due to the longer stand-off period. Finally, our $\mathrm{MP}+$ Blood and MP + HTK protocol were different from the clinical protocol. In the MP + Blood protocol, the heart grafts were perfused on our home-made $e x$ vivo perfusion circuit instead of the Transmedics Organ Care System, which has been currently used in the preservation of DCD hearts in the clinical setting. Additionally, as one of the crystalloid perfusates, the HTK solution has not yet been tested for the preservation of heart grafts with machine perfusion in our clinical setting.

\section{Conclusions}

Although compared with hypothermic continuous MP with histidine-tryptophan-ketoglutarate solution, normothermic continuous MP with autologous blood significantly upregulates the expression of ICAM-1 in the coronary artery, it significantly alleviates endothelial dysfunction of coronary artery of donor hearts in a porcine ex vivo model of donation following circulatory death. This protective effect is associated with the inhibition of apoptosis and nitro-oxidative stress in the coronary artery.

\section{Acknowledgments}

Funding: This research was supported by grants from Guangzhou Science and Technology Planning Project, No. 201804010067, Guangdong Science and Technology Planning Project, No. 2017ZC0064, and President Foundation of Nanfang Hospital, Southern Medical University (No. 2019c030 and No. 2017Z018).

\section{Footnote}

Reporting Checklist: The authors have completed the ARRIVE reporting checklist. Available at http://dx.doi.org/10.21037/ apm-20-131

Data Sharing Statement: Available at http://dx.doi. org/10.21037/apm-20-131

Conflicts of Interest: All authors have completed the ICMJE uniform disclosure form (available at http://dx.doi. org/10.21037/apm-20-131). The authors have no conflicts of interest to declare.

Ethical Statement: The authors are accountable for all aspects of the work in ensuring that questions related to the accuracy or integrity of any part of the work are appropriately investigated and resolved. All the healthy male pigs weighing 30-35 kg were used in this experiment and received care in compliance with the Guide for the Care and Use of Laboratory Animals (National Institutes of Health Publication No. 85-23, revised 1996). The study was approved by institutional ethics committee of Nanfang Hospital of Southern Medical University (No. NFEC2018-056).

Open Access Statement: This is an Open Access article distributed in accordance with the Creative Commons Attribution-NonCommercial-NoDerivs 4.0 International License (CC BY-NC-ND 4.0), which permits the noncommercial replication and distribution of the article with the strict proviso that no changes or edits are made and the original work is properly cited (including links to both the formal publication through the relevant DOI and the license). See: https://creativecommons.org/licenses/by-nc-nd/4.0/.

\section{References}

1. Lund LH, Edwards LB, Kucheryavaya AY, et al. The registry of the International Society for Heart and Lung Transplantation: thirty-first official adult heart transplant report--2014; focus theme: retransplantation. J Heart Lung Transplant 2014;33:996-1008.

2. Longnus SL, Mathys V, Dornbierer M, et al. Heart transplantation with donation after circulatory determination of death. Nat Rev Cardiol 2014;11:354-63.

3. White CW, Messer SJ, Large SR, et al. Transplantation of Hearts Donated after Circulatory Death. Front Cardiovasc Med 2018;5:8.

4. Messer S, Page A, Colah S, et al. Human heart transplantation from donation after circulatory-determined death donors using normothermic regional perfusion and cold storage. J Heart Lung Transplant 2018;37:865-9.

5. Noterdaeme T, Detry O, Hans MF, et al. What is the potential increase in the heart graft pool by cardiac 
donation after circulatory death? Transpl Int 2013;26:61-6.

6. Messer S, Page A, Axell R, et al. Outcome after heart transplantation from donation after circulatory-determined death donors. J Heart Lung Transplant 2017;36:1311-8.

7. Singhal AK, Symons JD, Boudina S, et al. Role of Endothelial Cells in Myocardial Ischemia-Reperfusion Injury. Vasc Dis Prev 2010;7:1-14.

8. Parolari A, Rubini P, Cannata A, et al. Endothelial damage during myocardial preservation and storage. Ann Thorac Surg 2002;73:682-90.

9. Méndez-Carmona N, Wyss RK, Arnold M, et al. Differential effects of ischemia/reperfusion on endothelial function and contractility in donation after circulatory death. J Heart Lung Transplant 2019;38:767-77.

10. Shreeniwas R, Koga S, Karakurum M, et al. Hypoxiamediated induction of endothelial cell interleukin-1 alpha. An autocrine mechanism promoting expression of leukocyte adhesion molecules on the vessel surface. J Clin Invest 1992;90:2333-9.

11. Ogawa S, Gerlach H, Esposito C, et al. Hypoxia modulates the barrier and coagulant function of cultured bovine endothelium. Increased monolayer permeability and induction of procoagulant properties. J Clin Invest 1990;85:1090-8.

12. Stoica SC, Goddard M, Large SR. The endothelium in clinical cardiac transplantation. Ann Thorac Surg 2002;73:1002-8.

13. Chew HC, Iyer A, Connellan M, et al. Outcomes of Donation After Circulatory Death Heart Transplantation in Australia. J Am Coll Cardiol 2019;73:1447-59.

14. Van Caenegem O, Beauloye C, Bertrand L, et al. Hypothermic continuous machine perfusion enables preservation of energy charge and functional recovery of heart grafts in an ex vivo model of donation following circulatory death. Eur J Cardiothorac Surg 2016;49:1348-53.

15. Koike N, Takeyoshi I, Ohki S, et al. The effect of shortterm coronary perfusion using a perfusion apparatus on canine heart transplantation from non-heart-beating donors. J Heart Lung Transplant 2003;22:810-7.

16. Scheule AM, Haas J, Zurakowski D, et al. A nonheart-beating donor model to evaluate functional and morphologic outcomes in resuscitated pig hearts. J Invest Surg 2002;15:125-35.

17. Rosenfeldt F, Ou R, Salamonsen R, et al. A novel combination technique of cold crystalloid perfusion but not cold storage facilitates transplantation of canine hearts donated after circulatory death. J Heart Lung Transplant
2016;35:1358-64.

18. Boyle EM, Jr., Pohlman TH, Cornejo CJ, et al. Endothelial cell injury in cardiovascular surgery: ischemiareperfusion. Ann Thorac Surg 1996;62:1868-75.

19. Tsao PS, Lefer AM. Time course and mechanism of endothelial dysfunction in isolated ischemic- and hypoxicperfused rat hearts. Am J Physiol 1990;259:H1660-6.

20. Schulz R, Kelm M, Heusch G. Nitric oxide in myocardial ischemia/reperfusion injury. Cardiovasc Res 2004;61:402-13.

21. Inserte J, Hernando V, Vilardosa U, et al. Activation of cGMP/protein kinase $\mathrm{G}$ pathway in postconditioned myocardium depends on reduced oxidative stress and preserved endothelial nitric oxide synthase coupling. J Am Heart Assoc 2013;2:e005975.

22. Vasquez-Vivar J. Tetrahydrobiopterin, superoxide, and vascular dysfunction. Free Radic Biol Med 2009;47:1108-19.

23. Otani H. The role of nitric oxide in myocardial repair and remodeling. Antioxid Redox Signal 2009;11:1913-28.

24. Yang BC, Nichols WW, Mehta JL. Cardioprotective Effects of Red Blood Cells on Ischemia and Reperfusion Injury in Isolated Rat Heart: Release of Nitric Oxide as a Potential Mechanism. J Cardiovasc Pharmacol Ther 1996;1:297-306.

25. Kupatt C, Weber C, Wolf DA, et al. Nitric oxide attenuates reoxygenation-induced ICAM-1 expression in coronary microvascular endothelium: role of NFkappaB. J Mol Cell Cardiol 1997;29:2599-609.

26. Niederberger P, Farine E, Arnold M, et al. High preischemic fatty acid levels decrease cardiac recovery in an isolated rat heart model of donation after circulatory death. Metabolism 2017;71:107-17.

27. Iyer A, Chew HC, Gao L, et al. Pathophysiological Trends During Withdrawal of Life Support: Implications for Organ Donation After Circulatory Death. Transplantation 2016;100:2621-9.

28. Vinten-Johansen J, Zhao ZQ, Nakamura M, et al. Nitric oxide and the vascular endothelium in myocardial ischemiareperfusion injury. Ann N Y Acad Sci 1999;874:354-70.

29. Lefer DJ, Flynn DM, Phillips ML, et al. A novel sialyl LewisX analog attenuates neutrophil accumulation and myocardial necrosis after ischemia and reperfusion. Circulation 1994;90:2390-401.

30. Sutherland FJ, Hearse DJ. The isolated blood and perfusion fluid perfused heart. Pharmacol Res 2000;41:613-27.

31. Walters HL, 3rd, Digerness SB, Naftel DC, et al. The 
response to ischemia in blood perfused vs. crystalloid perfused isolated rat heart preparations. J Mol Cell Cardiol 1992;24:1063-77.

32. Chew HC, Macdonald PS, Dhital KK. The donor heart and organ perfusion technology. J Thorac Dis
2019;11:S938-45.

33. Korkmaz-Icoz S, Li S, Huttner R, et al. Hypothermic perfusion of donor heart with a preservation solution supplemented by mesenchymal stem cells. J Heart Lung Transplant 2019;38:315-26.

Cite this article as: Zhou $\mathrm{P}, \mathrm{Liu} \mathrm{X}, \mathrm{Xiao} \mathrm{Z}, \mathrm{Xu} \mathrm{R}, \mathrm{Lin} \mathrm{X}$, Zhu P, Lu J, Zheng S. Machine perfusion of donor heart with normothermic blood versus hypothermic HTK in preserving coronary endothelium in a porcine model of DCD. Ann Palliat Med 2020;9(4):1476-1487. doi: 10.21037/apm-20-131 